\title{
Effects of Wetland Presence and Upland Land Use on Wild Hymenopteran and Dipteran Pollinators in the Rainwater Basin of Nebraska, USA
}

\author{
Angela Begosh $^{1} \cdot$ Loren M. Smith ${ }^{1} \cdot$ Cynthia N. Park ${ }^{2} \cdot$ Scott T. Mcmurry ${ }^{1} \cdot$ Ted G. Lagrange $^{3}$
}

Received: 11 July 2019 / Accepted: 6 November 2019 / Published online: 26 December 2019

(C) The Author(s) 2019

\begin{abstract}
Pollination is an ecosystem service that is in jeopardy globally. Decreasing trends in wild pollinator populations are primarily attributed to habitat loss and degradation. These concerns are especially apparent in the Rainwater Basin of Nebraska, USA where more than $90 \%$ of the land has been cultivated and $90 \%$ of the wetlands have been lost. We compared hymenopteran and dipteran pollinator abundance, richness, and diversity in 28 wetlands and their adjacent uplands within 3 dominant land-condition types: cropped, restored, and reference state. We used vane traps to capture local pollinator insects and sweep nets to collect insect pollinators on flowers. Vane-trap captures revealed a greater abundance, richness, and diversity of hymenopteran pollinators in uplands as compared to wetlands over the entire growing season in all land-condition types. However, net collections showed that hymenopterans foraged more in wetlands than in uplands, especially in restored wetlands. The exception was September when hymenopterans were captured in uplands more than wetlands while feeding on late season forbs. Dipteran vane-trap and sweepnet captures primarily consisted of hoverflies (Syrphidae). Hoverflies were more abundant in wetlands than uplands. All pollinators used both wetlands and uplands for resources. Thus, insect-pollinator conservation can be enhanced by promoting native forb communities and pollinator habitat through wetland restoration and the planting of buffer strips.
\end{abstract}

Keywords Diptera $\cdot$ Ecosystem services $\cdot$ Hymenoptera $\cdot$ Pollinators $\cdot$ Restoration ecology

\section{Introduction}

Global pollinator declines can jeopardize agricultural production as well as wild-plant populations and their associated biota (Potts et al. 2010). There are myriad issues that contribute to pollinator declines in wild pollinator populations. Interrelated factors include pesticides, pathogens, exotic species, climate change, and habitat loss and degradation (Biesmeijer et al. 2006; Winfree et al. 2009; Potts et al. 2010). In this paper, we focus on habitat loss and degradation,

Angela Begosh

angie.begosh@okstate.edu

1 Department of Integrative Biology, Oklahoma State University, Stillwater, OK 74059, USA

2 Coos Watershed Association, Coos Bay, OR, USA

3 Nebraska Game and Parks Commission, Lincoln, NE, USA which is the primary threat to native bee populations (Winfree et al. 2009).

Although the domestic honeybee (Apis mellifera L.) is the most important commercial pollinator (Calderone 2012b), research has documented that wild pollinators are more effective pollinators of several important crops such as sweet cherry (Holzschuh et al. 2012; Eeraerts et al. 2017), almonds (Alomar et al. 2018), apples (Mallinger and Gratton 2015), and lowbush blueberries (McKechnie et al. 2017). Furthermore, honeybee pollination is often supplementary to that of wild pollinators (Garibaldi et al. 2013; Eeraerts et al. 2017).

Bees are recognized as the primary pollinators (Willmer 2011), however, the importance of dipterans, especially nonSyrphid dipterans (Orford et al. 2015), is often overlooked (Larson et al. 2001). Many are major contributors to pollination services in agriculture and biodiversity, and are present in nearly all habitats (Ssymank et al. 2008). There is evidence that some populations of syrphids are in decline in North America, likely due to displacement by non-native species (Skevington et al. 2019). However, dipterans have different 
ecological requirements than wild bees, and could provide pollination service "insurance" in habitats where bees are threatened (Kearns 2001; Orford et al. 2015).

We conducted this study in one of the most productive cropland regions in the world, the Rainwater Basin (RWB) located in the Northern High Plains (Smith et al. 2011) (Fig. 1). One of the key features of this region, playa wetlands (hereafter referred to simply as "playas") are a crucial component of the Northern High Plains because they provide ecosystem services such as floodwater attenuation, recharging of the Ogallala aquifer, water-quality improvement, and biodiversity support (Haukos and Smith 2003; Smith et al. 2011). However, North American grasslands have declined by as much as $99 \%$, largely due to cultivation, and less than $10 \%$ of the historically present playas are estimated to remain in the RWB (Samson and Knopf 1994; LaGrange 2005; RWBJV, 2018).

However, more than $90 \%$ of the RWB has been cultivated and $90 \%$ of the region's playas have been converted to other land-condition types (). Cultivating watersheds increases erosion and other runoff, and sacrifices floral diversity in uplands and downslope wetland communities (O'Connell et al. 2012), consequently influencing the abundance and diversity of plants for pollinating insects.

Wild pollinators need abundant and diverse floral resources (Kwaiser and Hendrix 2007; Fründ et al. 2010), and nesting habitat that includes areas of bare soil for ground-nesting bees and pithy material for stem nesters (Potts et al. 2005; Black et al. 2011). Proximity to natural or semi-natural areas has shown to increase abundance and species richness of wild pollinators (Garibaldi, 2011; Smith et al. 2013; Alomar, 2018). Native landscapes historically provided a diversity of floral resources that sustained diverse mutualistic pollinator-plant relationships (Potts et al. 2010). Although some generalists can survive in a changing habitat, many native species of pollinators with more specialized requirements cannot (Kwaiser and Hendrix, 2007; Aizen et al. 2012). If habitat quality degrades and homogenization of the landscape becomes significant, an ecosystem may suffer from a loss of pollinator species richness (Mogren et al., 2016) and consequently, pollination services. Most invertebrate pollinator species successfully reproduce if their habitat provides nest sites, building materials, and food plants in close proximity and therefore, these remaining pieces of suitable habitat may be essential for pollination in the region (James and Pitts-Singer 2008; Hoffman Black et al. 2011). Wetlands have been demonstrated to be a landscape feature associated with increased pollinator abundance and diversity (Evans et al. 2018). Therefore, our research focused on how the presence, restoration, and surrounding land use of playa systems in the RWB can influence pollinator populations in this highly altered landscape.

The purpose of our research was:

- to compare abundance, richness, and diversity among the major land-condition types in the RWB; cropland, restored, and reference;

- to compare abundance, richness, and diversity of hymenopteran and dipteran pollinators in playas and their adjacent uplands;

- determine if playas serve as important habitat for hymenopteran and dipteran pollinators;

- provide management recommendations based on our results.
Fig. 1 Rainwater Basin, Nebraska, USA where hymenoptera and dipterans were collected in 2014 and 2015. Map courtesy of the Rainwater Basin Joint Venture (Rainwater Basin Joint Venture 2019)

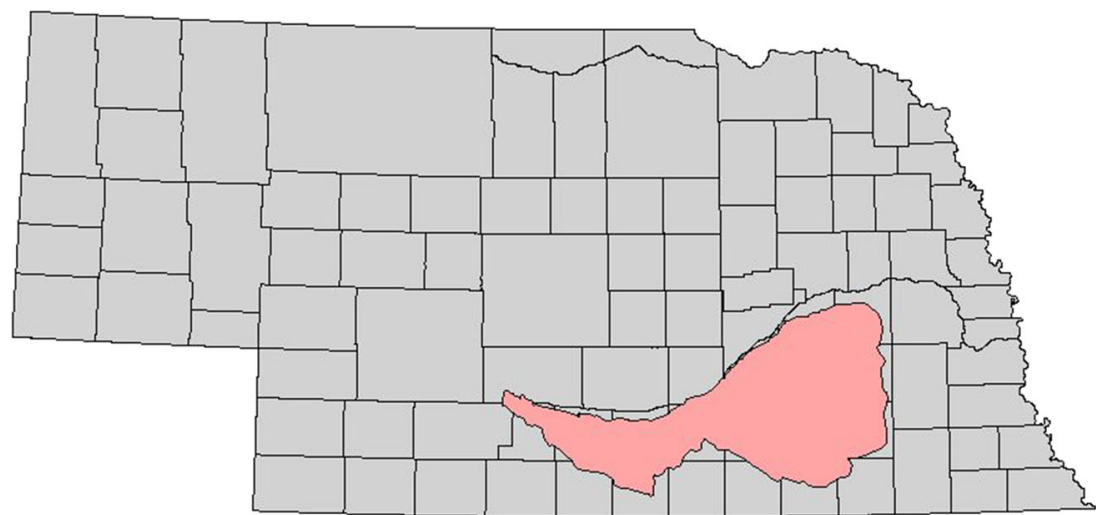




\section{Materials and Methods}

\section{Study Area}

We studied playas and their adjacent upland watersheds within three land-condition types: cropped, restored, and reference. This design allowed us to evaluate the effect of restoration practices on native pollinators. Reference sites were the best representation of the original landscape that we could find. The RWB comprises approximately $15,907 \mathrm{~km}^{2}$ in southcentral Nebraska (LaGrange 2005) (Fig. 1). Historically, the landscape was flat to gently rolling plains with abundant natural playas that held rain and runoff water (LaGrange et al. 2011). Grasslands in this region consisted of mixed grasses including bluestems (Andropogon spp.), wheatgrasses (Agropyron spp.), and needle grasses (Stipa spp.). Tallgrass plant communities that included bluestems along with switchgrass (Panicum virgatum), and Indian grass (Sorghastum nutans) occurred in the extreme eastern portion of the region (Kuchler 1964).

Restored sites were enrolled in the United States Department of Agriculture's Wetlands Reserve Program (WRP), now known as the Agricultural Conservation Easement Program's Wetland Reserve Easements (WRE). While conservation programs that focus on upland restoration are rare in the RWB, wetland restoration programs, such as the WRP, are prominent (Smith et al. 2011). This program offers financial and technical support to landowners for enhancing, restoring, or protecting wetlands and the immediate watershed (NRCS 2008a). Conservation practices included planting a filter strip of perennial cover to reduce sedimentation and nutrient runoff, filling of reuse pits (an excavation constructed to capture irrigation water for reuse (Rainwater Basin Joint Venture 2019)), and removal of sediment to improve hydrology and plant communities (Gleason et al. 2011; Beas et al. 2013). Even with the great loss of playas, the RWB is a focal point of the Central Flyway that over 170 different species of birds rely on (NRCS 2008b). Consequently, RWB conservation primarily focuses on providing wetland habitat and critical food resources for midcontinent migrating waterfowl (Webb et al. 2010). State and federal wildlife agencies have conserved over 2500 ha of playas since the 1960s (Walker 2016). Over $75 \%$ of the conserved area is protected in the form of federal Waterfowl Production Areas, state Wildlife Management Areas, and conservation easements such as those obtained through the WRP (Grosse 2014). Therefore, remaining playa wetlands and their surrounding uplands in the RWB may be important as pollinator habitat in this highly altered landscape.

Currently, the RWB has been overwhelmingly converted to soybean (Glycine max) and corn (Zea mays) production (Smith 2003). We selected cropland sites based on presence of a playa within an active cropping operation and willingness of the landowner to cooperate.

\section{Field Sampling}

Insects were collected from the first week of April until midOctober in 2014 and 2015 in the RWB. Twenty-eight different sites were sampled 14 times (once every 2 weeks for 7 months) in 2014 and 2015. Each sampling site featured a playa that was embedded within one of three different land-condition types: cropped $(n=10)$, restored $(n=9)$, and reference $(n=$ 9). At each site, we selected 6 random sampling points; 3 within the playa and 3 within the adjacent uplands immediate to the playa (Fig. 2). We collected insects with vane traps (SpringstarTM LLC, Woodinville, WA, USA) and insect nets (Stephen and Rao 2005; Stephen and Rao 2007). Vane traps consisted of a bright yellow plastic jar fitted with a blue fabricated polypropylene screw cap funnel into which two, blue, cross vanes were inserted (Stephen and Rao 2007). A wooden stake was placed at each sampling point and a trap was attached with wire so it was visible in the surrounding vegetation. Upland points were established at least $25 \mathrm{~m}$ away from the playa edge so that they were located within the upland plant community. There was no maximum distance as it varied based on the vegetation characteristics of the playa watershed. Traps were set up between 0900 and 1100 CDT at different sites each day and then picked up the following morning during the same time-period, allowing for approximately 24-h exposure. We then replaced the funnel with a lid and the jars were placed in a freezer to kill captured specimens.

We used 30.5-cm-diameter insect nets for targeted netting. We established a 25-m-long, 1.0-m-wide transect belt at each vane trap location and positioned it so the wooden stake with the trap was at the midline of the belt $(12 \mathrm{~m}$ up the transect with $0.5 \mathrm{~m}$ on either side). Technicians scanned the belt transect for $6 \mathrm{~min}$, observing flowers and captures insects when they fed on or manipulated flowers within the transect belt. We paused the timer while the insect was placed in a killing jar of ethyl acetate. Once it was dead, we placed the captured insect in a plastic sample bag labeled with site, transect number, date, and species of the flower on which it was captured. Targeted sampling started at approximately 1100 CDT, after the vane traps for the day's sites had been set out.

\section{Insect Identification}

All specimens were kept in a freezer at $-10{ }^{\circ} \mathrm{C}$ until they were identified. Insect specimens were first identified to order by simple observation from a local entomologist and then to genera and species using taxonomic keys as needed. A complete list of taxonomic keys and identification methods used can be found in Park et al. (2017). Because Hymenoptera and Diptera are the two most important pollinator orders in North America, we focused on these two orders (Inouye et al. 2015; Orford, 2015). 
Fig. 2 Aerial imagery of a reference site, Alberding Lagoon National Wildlife Management Area (WMA), Clay County, Nebraska (Google Earth, earth. google.com/web/). The WMA is rangeland with a playa located in the middle. Three traps were placed in the playa (represented by triangle points) and three traps in the upland (represented by square points). The upland traps were placed at a minimum of $25 \mathrm{~m}$ from the playa edge with goal of placing the trap in an upland plant community that differs from the playa plant community. Triangles represent the playa traps and squares represent the upland traps. The playa traps were pooled at each site to make a playa sampling unit and the upland traps were pooled to make an upland sampling unit

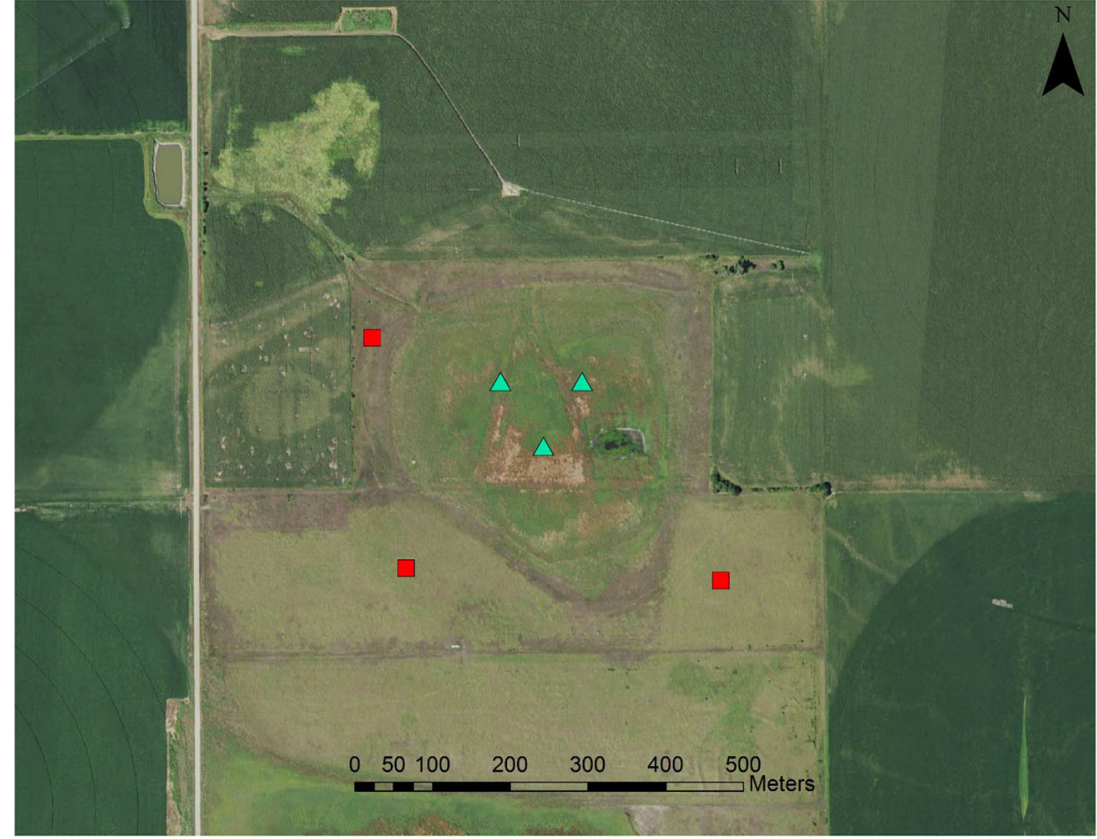

Specimens of additional insect orders were collected, including Coleoptera and Lepidoptera, but we did not include those orders in this analysis (see Park et al. 2017 for complete list). We used Willmer (2011) to determine the families of Hymenoptera and Diptera recognized as pollinators. We used Triplehorn et al. (2005) to confirm dipteran life history and anthophilous behavior, recognizing that pollinators in one environment may only be anthophilous in another habitat, or potential pollinators.

\section{Statistical Analyses}

We measured abundance, richness, and diversity (ShannonWeiner index) of hymenopteran and dipteran pollinators among land-condition types and landscape positions. We used a subset of data from Park et al. (2017). Insects collected in the 3 vane traps in the playas and the 3 vane traps in the uplands were pooled to create a playa and upland sampling unit for each site per sampling period. We performed an analysis of variance (ANOVA) (Minitab 17 Statistical Software, State College, PA) to compare the main effects and interactions of biweekly sampling period, land-condition type (cropped, restored, reference) and landscape position (playa, upland), for insect vane trap and net-sampling techniques. Land-condition type, land position, and sampling unit were classified as fixed effects and year was a random effect. When significant relationships occurred we used Tukey's pairwise comparisons to examine differences in means. We used an alpha of 0.1 because we wanted to detect large-scale biological effects if they exist.

\section{Results}

\section{Hymenoptera}

Hymenopterans comprised almost $80 \%$ of all specimens caught. Over 86,500 bees from five different families were collected. Apidae and Halictidae made up 98\% (62\% and $36 \%$, respectively) of the hymenopteran samples collected. Additional families included Andrenidae, Colletidae, and Megachilidae. Apidae was the most species rich family with 55 total species, including 11 Bombus and 16 Melissodes species. The 16 Melissodes species made up $76 \%$ of all specimens collected within Apidae. Less than $2 \%$ of.

Hymenopterans caught were not bees; wasps and ants each equaled $1 \%$ of total insects.

Two bee families, Andrenidae and Megachilidae, and the wasp family Vespidae had greater representation in net collections than traps. However, Halictidae was greater in trap than net collections and Apidae and Colletidae frequencies were not different between the two sampling techniques.

With vane traps, reference uplands $(\bar{x}=70.67, \mathrm{SE}=8.28)$ had higher hymenopteran abundance than crop uplands $(x=$ 58.06, $\mathrm{SE}=6.8,[\mathrm{~F}(2,1420)=4.06, p=0.017]$ (Fig. 3a). Hymenoptera abundance was higher in early September (pe$\operatorname{riod} 12)(x=166.17, \mathrm{SE}=21.15),[\mathrm{F}(13,1420)=29.03, \mathrm{p}<<$ $0.001]$ (a) than all other periods $10(x=112.10, \mathrm{SE}=13.73)$ (b), $9(x=92.56, \mathrm{SE}=10.35)(\mathrm{bc}), 11(x=83.32, \mathrm{SE}=8.07)$ (bcd), $8(x=77.44, \mathrm{SE}=6.55)($ bcde $), 13(x=68.14, \mathrm{SE}=6.56)$ (cdef), 5 ( $x=50.37, \mathrm{SE}=7.96)(\operatorname{defg}), 4(x=38.87, \mathrm{SE}=9.68)$ (efgh), $7(x=34.53, \mathrm{SE}=3.29)(\mathrm{fgh}), 6(x=29, \mathrm{SE}=4.11)$ (fgh), $14(x=24.68, \mathrm{SE}=4.73)(\mathrm{gh}), 3(x=9.34, \mathrm{SE}=2.20)$ 
a

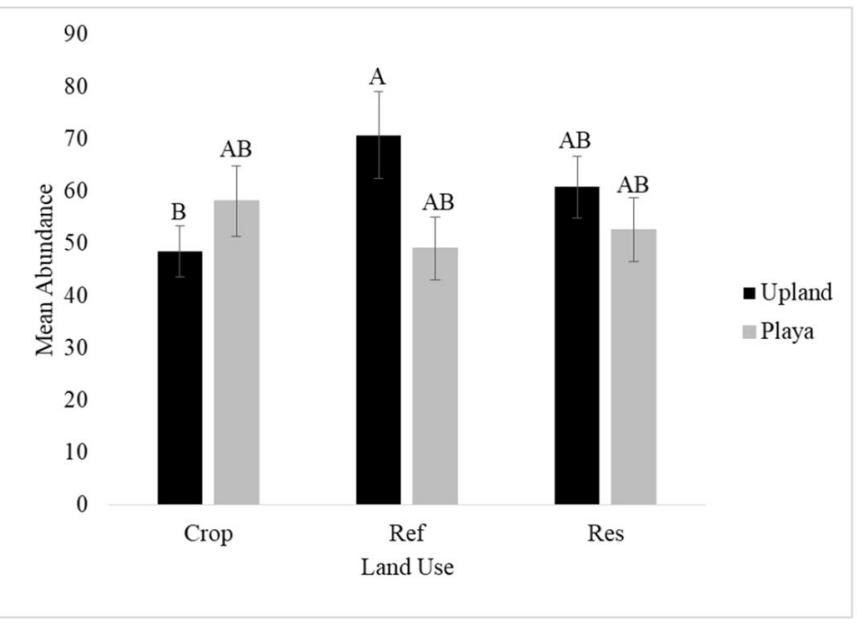

$\mathrm{c}$

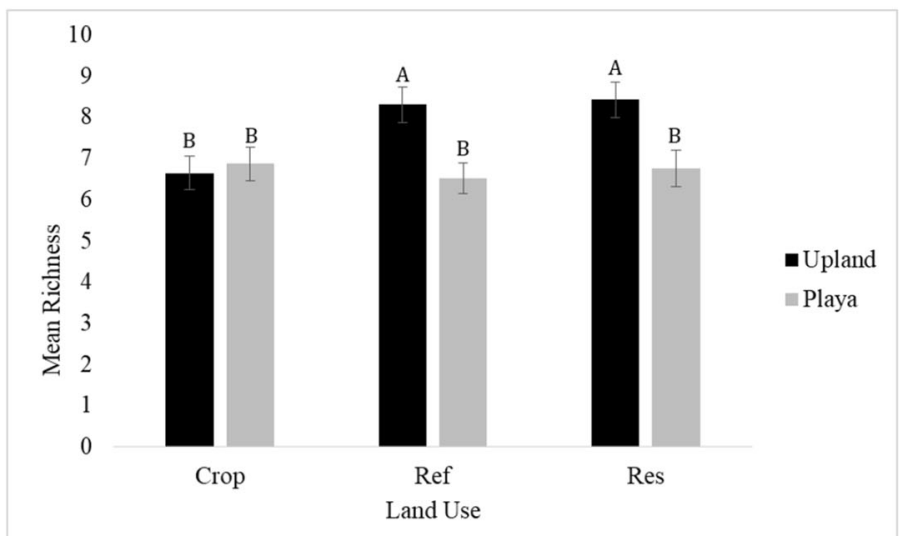

b

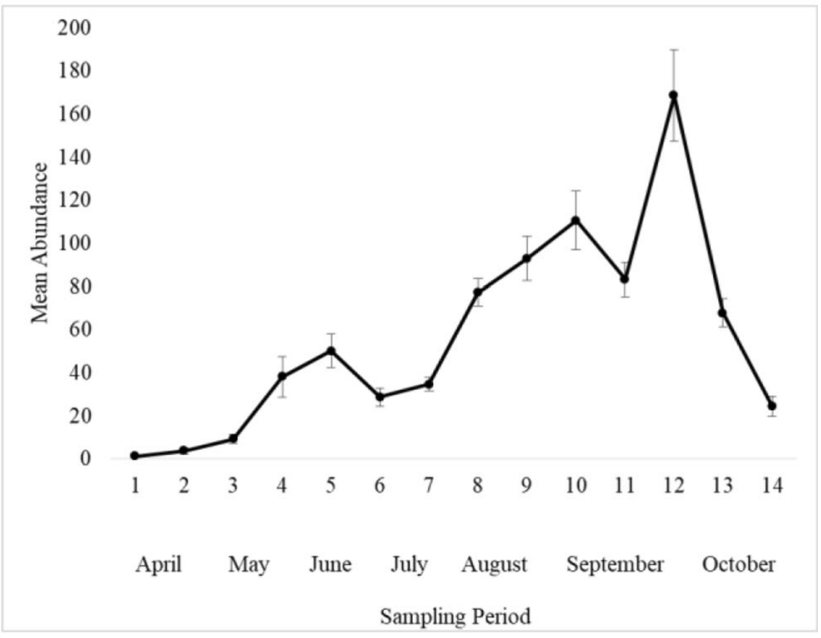

d

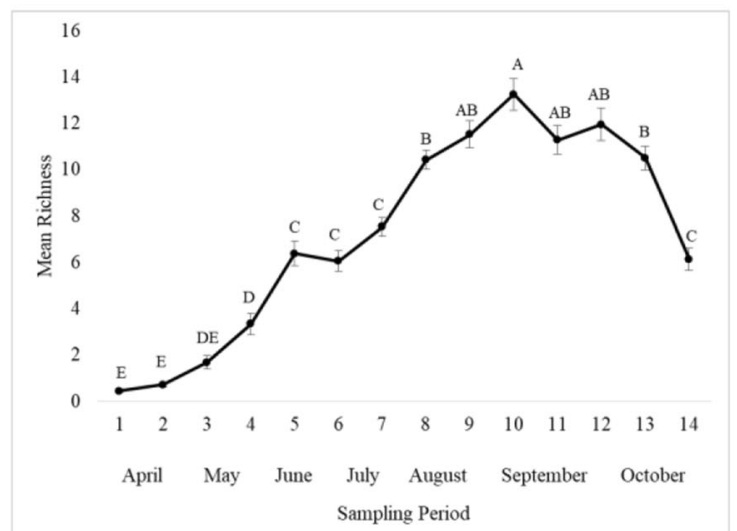

Fig. 3 Differences in Hymenoptera abundance and richness from blue vane trap samples collected in the Rainwater Basin of Nebraska in 2014 and 2015. a Abundance among three land-use conditions and two landscape positions. Means that do not share a letter are significantly different. b Abundance over 14 sampling periods. c Richness among

$(\mathrm{gh}), 2(x=3.71, \mathrm{SE}=1.55)(\mathrm{h}), 1(x=1.3, \mathrm{SE}=0.41),[\mathrm{F}(13$, 1420) $=29.35, \mathrm{p}=<0.001)$ (h) (Fig. 3b) (Table 1). Tukey's post hoc results are indicated in parentheses when not included in the accompanying figures throughout this section.

Hymenopteran richness was higher in restored $(x=8.41$, $\mathrm{SE}=0.44)$ and reference uplands $(x=8.29, \mathrm{SE}=0.43)$ than crop uplands $(\bar{x}=6.63, \mathrm{SE}=0.40)$, crop playas $(\bar{x}=6.86$, $\mathrm{SE}=0.40)$, restored playas $(x=6.76, \mathrm{SE}=0.44)$, and reference playas $(\bar{x}=6.76, \mathrm{SE}=0.44),[\mathrm{F}(2,1420)=7.06, p=$ 0.001 ] (Fig. 3c). Hymenopteran richness in early August ( $x$ $=13.31, \mathrm{SE}=0.70)$ was higher than in all other sampling periods except early September $(x=11.87, \mathrm{SE}=0.72)$ and late July $(x=10.46, \mathrm{SE}=0.41),[\mathrm{F}(13,1420)=94.94, \mathrm{p}<<0.001)$ (Fig. 3d) (Table 1).

Hymenoptera diversity in restored $(x=1.27, \mathrm{SE}=0.05)$ (a) and reference $(x=1.23, \mathrm{SE}=0.05)$ (a) uplands was higher than in crop uplands $(x=1.03, \mathrm{SE}=0.05) \mathrm{b})$, reference playas $(\bar{x}$ interaction of land-condition*land position. d Richness over 14 sampling periods. Means that do not share a letter are significantly different. Crop $=$ cropland, Ref $=$ reference and $\mathrm{Res}=$ restored land use conditions. Bars indicate standard error

$=1.08, \mathrm{SE}=0.05)(\mathrm{b})$, restored playas $(x=1.05, \mathrm{SE}=0.06)(\mathrm{b})$, and crop playas $(x=1.04, \mathrm{SE}=0.05)(\mathrm{b}),[\mathrm{F}(2,1420)=6.35$, $p=0.002$. Hymenopteran diversity was highest in sampling periods $10(x=1.75, \mathrm{SE}=0.05)(\mathrm{a}), 9(x=1.67, \mathrm{SE}=0.07)$ $(\mathrm{ab}), 11(x=1.66, \mathrm{SE}=0.05)(\mathrm{ab}), 8(x=1.65, \mathrm{SE}=0.04)$ $(\mathrm{ab})$, and $13(x=1.63, \mathrm{SE}=0.05)(\mathrm{abc}),[\mathrm{F}(13,1420)=$ 139.26, $p<0.001$ ] (Table 1).

Hymenoptera comprised $26 \%$ of insects collected with nets; $93 \%$ were bees. Three hundred seventy-five bees from five different families (Andrenidae, Apidae, Colletidae, Halictidae, and Megachilidae) were caught on flowers. Apidae had the highest abundance with Bombus and Melissodes being the most common genera. Twenty-nine wasps from six families were collected. Vespidae had the most individuals overall, comprising over half of the wasps caught.

Based on targeted netting data, Hymenoptera abundance in restored playas in period $11(x=3.06, \mathrm{SE}=1.04)$ (a) were 
Table 1 Abundance, species richness, and diversity of Hymenoptera collected with vane traps in the Rainwater Basin of Nebraska during the growing seasons of 2014 and 2015

\begin{tabular}{|c|c|c|c|c|c|}
\hline Source & df & Adj SS & Adj MS & $\begin{array}{l}\mathrm{F}- \\
\text { value }\end{array}$ & $P$ value \\
\hline \multicolumn{6}{|l|}{ Abundance } \\
\hline Land-condition & 2 & 21451 & 10726 & 1.34 & 0.263 \\
\hline County & 7 & 185538 & 26505 & 3.30 & 0.002 \\
\hline Period & 13 & 3063164 & 235628 & 29.35 & $<0.001$ \\
\hline Position & 1 & 15284 & 15284 & 1.90 & 0.168 \\
\hline Year & 1 & 698559 & 698559 & 87.03 & $<0.001$ \\
\hline Land-condition*Period & 26 & 263137 & 10121 & 1.26 & 0.172 \\
\hline Land-condition*Position & 2 & 65193 & 32597 & 4.06 & 0.017 \\
\hline Position*Period & 13 & 71726 & 5517 & 0.69 & 0.777 \\
\hline Land-condition*Position*Period & 26 & 108327 & 4166 & 0.52 & 0.979 \\
\hline Error & 1420 & 11398446 & 8027 & & \\
\hline Lack of fit & 944 & 9389605 & 9947 & 2.36 & $<0.001$ \\
\hline Pure error & 476 & 2008841 & 4220 & & \\
\hline Total & 1511 & 15871702 & & & \\
\hline \multicolumn{6}{|l|}{ Richness } \\
\hline Land-condition & 2 & 266.8 & 133.40 & 6.20 & 0.002 \\
\hline County & 7 & 771.2 & 110.17 & 5.12 & $<0.001$ \\
\hline Period & 13 & 27074.1 & 2082.62 & 96.86 & $<0.001$ \\
\hline Position & 1 & 426.8 & 426.85 & 19.85 & $<0.001$ \\
\hline Year & 1 & 5611.7 & 5611.70 & 260.99 & $<0.001$ \\
\hline Land-condition*Period & 26 & 572.9 & 22.03 & 1.02 & 0.429 \\
\hline Land-condition*Position & 2 & 335.7 & 167.84 & 7.81 & $<0.001$ \\
\hline Position*Period & 13 & 149.7 & 11.51 & 0.54 & 0.904 \\
\hline Land-condition*Position*Period & 26 & 294.0 & 11.31 & 0.53 & 0.976 \\
\hline Error & 1420 & 30532.7 & 21.50 & & \\
\hline Lack of fit & 944 & 24252.5 & 25.69 & 1.95 & $<0.001$ \\
\hline Pure error & 476 & 6280.2 & 13.19 & & \\
\hline Total & 1511 & 66202.4 & & & \\
\hline \multicolumn{6}{|l|}{ Diversity } \\
\hline Land-condition & 2 & 5.10 & 2.5479 & 8.94 & $<0.001$ \\
\hline County & 7 & 11.85 & 1.6926 & 5.94 & $<0.001$ \\
\hline Period & 13 & 515.84 & 39.6798 & 139.26 & $<0.001$ \\
\hline Position & 1 & 5.73 & 5.7293 & 20.11 & $<0.001$ \\
\hline Year & 1 & 50.84 & 50.8368 & 178.41 & $<0.001$ \\
\hline Land-condition*Period & 26 & 7.90 & 0.3040 & 1.07 & 0.373 \\
\hline Land-condition*Position & 2 & 3.62 & 1.8086 & 6.35 & 0.002 \\
\hline Position*Period & 13 & 3.69 & 0.2838 & 1.00 & 0.452 \\
\hline Land-condition*Position*Period & 26 & 4.88 & 0.1877 & 0.66 & 0.904 \\
\hline Error & 1420 & 404.61 & 0.2849 & & \\
\hline Lack of fit & 944 & 306.87 & 0.3251 & 1.58 & $<0.001$ \\
\hline Pure error & 476 & 97.74 & 0.2053 & & \\
\hline Total & 1511 & 1021.03 & & & \\
\hline
\end{tabular}

ANOVA analyses were used to compare three fixed factors (land-condition type, landscape position, and sampling period) and two random factors (year and county) similar to reference playas $(\bar{x}=1.67, \mathrm{SE}=0.54)(\mathrm{abcd})$ and higher than cropland wetlands $(x=1.3, \mathrm{SE}=0.54)(\mathrm{bcd})$ restored uplands $(x=0.53, \mathrm{SE}=0.27)(\mathrm{cd})$, reference uplands $(x$
$=0.17, \mathrm{SE}=0.09)(\mathrm{d})$, and cropland uplands $(\bar{x}=0.05, \mathrm{SE}=$ $0.05)(\mathrm{d}),[\mathrm{F}(26,1448)=1.77, p=0.010]$ in the same sampling period. Within sampling period 10 , restored playas had higher 
abundance $(\bar{x}=2.88, \mathrm{SE}=1.47)(\mathrm{ab})$ than reference playas $(\bar{x}$ $=0.5, \mathrm{SE}=0.16)(\mathrm{d})$, restored uplands $(x=0.35, \mathrm{SE}=0.17)(\mathrm{d})$, reference uplands $(x=0.28, \mathrm{SE}=0.16)(\mathrm{d})$, cropland uplands $(x$ $=0.1, \mathrm{SE}=0.1)(\mathrm{d})$ and cropland playas $(x=0.1, \mathrm{SE}=0.07)(\mathrm{d})$, $[\mathrm{F}(26,1448)=1.77, \mathrm{p}=0.010]$. Reference uplands in period $12(x=2.39, \mathrm{SE}=1.49)$ had higher abundance than restored playas $(x=0.12, \mathrm{SE}=0.08)$ and cropland uplands $(x=0.100$, $\mathrm{SE}=0.07)$, and were similar to restored uplands $(x=1.18, \mathrm{SE}=$ $0.49)$ (bcd), reference playas $(x=1.0, \mathrm{SE}=0.47)(\mathrm{cd})$, and cropland playas $(x=0.95, \mathrm{SE}=0.54)(\mathrm{cd}),[\mathrm{F}(26,1455)=1.75, p=$ 0.011] within the sampling period (Fig. 4a) (Table 2).

In sampling period 11, Hymenoptera richness in restored playas $(x=1.82, \mathrm{SE}=0.48)(\mathrm{a})$, was higher in all sampling units except reference playas $(\bar{x}=1.22, \mathrm{SE}=0.38)(\mathrm{ab})$. Reference playas had similar abundance to cropland playas $(x=0.7, \mathrm{SE}=0.24)$ (bcde), but had higher abundance than restored $(x=0.41, \mathrm{SE}=0.21)(\mathrm{cde})$, reference $(x=0.17, \mathrm{SE}=$ $0.09)$ (cde), and cropland uplands $(x=0.05, \mathrm{SE}=0.05)(\mathrm{de}),[\mathrm{F}$ $(26,1448)=1.67, p=0.019]$. In sampling period 10 , restored playas $(x=0.88, \mathrm{SE}=0.04)(\mathrm{bc})$ had higher richness than croplands playas $(x=0.10, \mathrm{SE}=0.07)(\mathrm{de})$ and cropland uplands $(x$
$=0.10, \mathrm{SE}=0.1)(\mathrm{de})$, but was similar to reference playas $(\bar{x}$ $=0.33, \mathrm{SE}=0.16)(\mathrm{cde})$, restored uplands $(x=0.29, \mathrm{SE}=0.14)$ (cde), and reference uplands $(x=0.22, \mathrm{SE}=0.13)($ cde $),[\mathrm{F}$ $(26,1448)=1.67, \mathrm{p}=0.019]$ (Fig. 4b) (Table 2).

Restored playas in period $11(x=0.52, \mathrm{SE}=0.16)(\mathrm{a}) \mathrm{had}$ higher diversity than reference $(x=0.29, \mathrm{SE}=0.12)(\mathrm{b})$ and cropland playas $(x=0.19, \mathrm{SE}=0.07)(\mathrm{bcd})$, and restored $(x$ $=0.10, \mathrm{SE}=0.07)(\mathrm{bcd})$, reference $(x=0.0, \mathrm{SE}=0.0)(\mathrm{d})$, and cropland uplands $(x=0.0, \mathrm{SE}=0.0)(\mathrm{d}),[\mathrm{F}(26,1448)=1.92$, $p=0.004]$ within the sampling period. In period 12 , restored uplands $(x=0.25, \mathrm{SE}=0.11)(\mathrm{bc})$ had higher diversity than restored playas $(x=0, \mathrm{SE}=0.0)(\mathrm{d})$ and cropland uplands $(x$ $=0, \mathrm{SE}=0)(\mathrm{d})$, but were similar to reference uplands $(x=0.15$, $\mathrm{SE}=0.08)(\mathrm{bcd})$, cropland playas $(x=0.15, \mathrm{SE}=0.08)(\mathrm{bcd})$, and reference playas $(\bar{x}=0.11, \mathrm{SE}=0.06)(\mathrm{bcd}),[\mathrm{F}(26$, $1448)=1.92, \mathrm{p}=0.004]($ Table 2$)$.

\section{Diptera}

The second largest order represented was the flies, totaling 12,266 specimens and $12 \%$ of all insects collected in
Fig. 4 Differences in Hymenoptera abundance and richness from targeted net samples in the Rainwater Basin of Nebraska from targeted net collections in 2014 and 2015. a Three-way interaction showing Hymenoptera abundance of landcondition*landscape position over 14 sampling periods. b Three-way interaction showing Hymenoptera richness of landcondition*landscape position over 14 sampling periods. Crop $=$ cropland, Ref $=$ reference and Res $=$ restored land-conditions . $\mathrm{Pla}=$ playa, $\mathrm{up}=$ upland land positions. Bars indicate standard error a

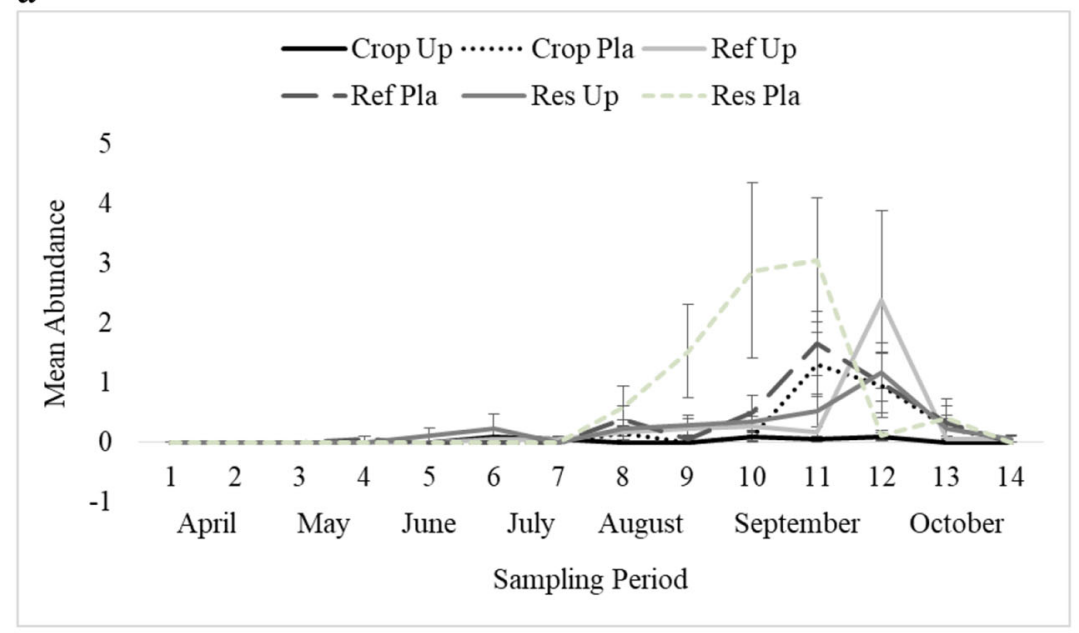

b

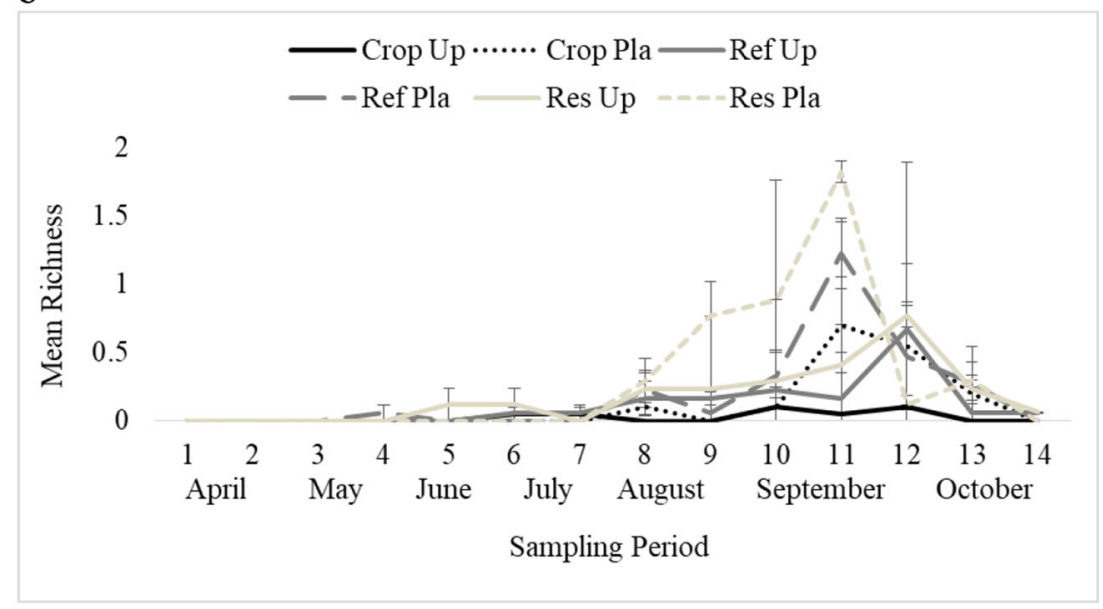


Table 2 Abundance, species richness, and diversity of Hymenoptera collected with net sampling in the Rainwater Basin of Nebraska during the growing seasons of 2014 and 2015

\begin{tabular}{|c|c|c|c|c|c|}
\hline Source & df & Adj SS & Adj MS & $\begin{array}{l}\mathrm{F}- \\
\text { value }\end{array}$ & $\mathrm{P}$ value \\
\hline \multicolumn{6}{|l|}{ Abundance } \\
\hline Land-condition & 2 & 34.95 & 17.4760 & 10.40 & $<0.001$ \\
\hline County & 7 & 28.84 & 4.1207 & 2.45 & 0.017 \\
\hline Period & 13 & 205.76 & 15.8273 & 9.42 & $<0.001$ \\
\hline Position & 1 & 13.19 & 13.1863 & 7.85 & 0.005 \\
\hline Year & 1 & 0.53 & 0.5284 & 0.31 & 0.575 \\
\hline Land-condition*Period & 26 & 93.99 & 3.6151 & 2.15 & 0.001 \\
\hline Land-condition*Position & 2 & 6.20 & 3.0999 & 1.84 & 0.158 \\
\hline Position*Period & 13 & 110.89 & 8.5297 & 5.08 & $<0.001$ \\
\hline Land-condition*Position*Period & 26 & 77.21 & 2.9695 & 1.77 & 0.010 \\
\hline Error & 1448 & 2433.63 & 1.6807 & & \\
\hline Lack of fit & 972 & 1545.13 & 1.5896 & 0.85 & 0.980 \\
\hline Pure error & 476 & 888.50 & 1.8666 & & \\
\hline Total & 1539 & 2980.02 & & & \\
\hline \multicolumn{6}{|l|}{ Richness } \\
\hline Land-condition & 2 & 9.761 & 4.88042 & 16.98 & $<0.001$ \\
\hline County & 7 & 5.290 & 0.75570 & 2.63 & 0.011 \\
\hline Period & 13 & 65.445 & 5.03420 & 17.52 & $<0.001$ \\
\hline Position & 1 & 3.186 & 3.18554 & 11.09 & 0.001 \\
\hline Year & 1 & 0.577 & 0.57671 & 2.01 & 0.157 \\
\hline Land-condition*Period & 26 & 14.523 & 0.55856 & 1.94 & 0.003 \\
\hline Land-condition*Position & 2 & 0.088 & 0.04417 & 0.15 & 0.858 \\
\hline Position*Period & 13 & 30.255 & 2.32734 & 8.10 & $<0.001$ \\
\hline Land-condition*Position*Period & 26 & 12.474 & 0.47977 & 1.67 & 0.019 \\
\hline Error & 1448 & 416.067 & 0.28734 & & \\
\hline Lack of fit & 972 & 313.900 & 0.32294 & 1.50 & $<0.001$ \\
\hline Pure error & 476 & 102.167 & 0.21464 & & \\
\hline Total & 1539 & 551.049 & & & \\
\hline \multicolumn{6}{|l|}{ Diversity } \\
\hline Land-condition & 2 & 0.7375 & 0.368735 & 13.95 & $<0.001$ \\
\hline County & 7 & 0.4203 & 0.060043 & 2.27 & 0.027 \\
\hline Period & 13 & 4.1769 & 0.321300 & 12.16 & $<0.001$ \\
\hline Position & 1 & 0.2313 & 0.231298 & 8.75 & 0.003 \\
\hline Year & 1 & 0.1212 & 0.121239 & 4.59 & 0.032 \\
\hline Land-condition*Period & 26 & 1.0797 & 0.041526 & 1.57 & 0.034 \\
\hline Land-condition*Position & 2 & 0.0021 & 0.001029 & 0.04 & 0.962 \\
\hline Position*Period & 13 & 2.5356 & 0.195042 & 7.38 & $<0.001$ \\
\hline Land-condition*Position*Period & 26 & 1.3168 & 0.050648 & 1.92 & 0.004 \\
\hline Error & 1448 & 38.2742 & 0.026432 & & \\
\hline Lack of fit & 972 & 29.3310 & 0.030176 & 1.61 & $<0.001$ \\
\hline Pure error & 476 & 8.9432 & 0.018788 & & \\
\hline Total & 1539 & 48.3651 & & & \\
\hline
\end{tabular}

ANOVA analyses were used to compare three fixed factors (land-condition type, landscape position, and sampling period) and two random factors (year and county) traps. Syrphidae comprised $65 \%$ of all flies collected, followed by Anthomyiidae with $21 \%$. Most of the other 29 families each made up less than $1 \%$ of all flies collected, and 13 families were not identified to a lower taxonomic level because they were not noted pollinators (Willmer 2011). 
With vane trap data, Diptera abundance differed by landcondition in period 3 . Restored sites $(x=20.97, \mathrm{SE}=8.75)$ (abc) had greater abundance than croplands $(x=1.7, \mathrm{SE}=$ $0.56)(\mathrm{h})$, and had similar abundance to reference sites $(x$ $=8.64, \mathrm{SE}=2.93)(\mathrm{abcdefg}),[\mathrm{F}(26,1420)=1.68, p=0.017]$ (Fig. 5a) (Table 3).

Sampling period was the primary driver of dipteran richness variation, and there were no significant differences among landcondition types within a sampling period. Richness increased in early summer and again in October with the maximum richness observed in sampling period 14 (Fig. 5b) (Table 3). Sampling period was also the primary driver with diversity, and it was not significantly different among land-condition types within the same sampling period (Table 3 ).

Diptera, totaling $32 \%$ of all net specimens caught, represented the largest order collected with nets. We collected 503 individuals from 14 families were collected. Syrphidae comprised the vast majority $(85 \%)$ of dipterans collected. Ten syrphid genera were identified. Eristalis and Helophilus had the highest abundance with $47 \%$ and $22 \%$, respectively.
Playa sites in period 11 were more abundant $(x=2.42, \mathrm{SE}=$ $0.61)$ (a) than upland sites in the same period $(x=0, \mathrm{SE}=0)$ (c), $[\mathrm{F}(13,1448)=5.62, \mathrm{p}=<0.001]$ (Fig. 6a) (Table 4). Restored sites had higher abundance $(x=2.42, \mathrm{SE}=0.61)$ (a) than cropland sites $(x=2.42, \mathrm{SE}=0.61)(\mathrm{c})$, and were similar to reference sites $(\bar{x}=2.42, \mathrm{SE}=0.61)(\mathrm{ab}),[\mathrm{F}(2,1448)=$ $0.00, p=0.00]$ (Fig. 6b) (Table 4).

Playas in period $11(x=1.36, \mathrm{SE}=0.3)$ (a) had higher richness than upland sites $(x=0.0, \mathrm{SE}=0.0)(\mathrm{c})$. Playas in period $10(\bar{x}=0.58, \mathrm{SE}=0.15)$ were also richer than upland sites in the same period $(\bar{x}=0.11, \mathrm{SE}=0.05),[\mathrm{F}(13$, $1448)=10.58,<0.001]($ Fig. 6c) (Table 4). Restored $(\bar{x}$ $=0.25, \mathrm{SE}=0.04)$ (a) and reference sites $(x=0.18, \mathrm{SE}=$ 0.03 ) (a) had higher richness than cropland sites $(x=0.09$, $\mathrm{SE}=0.02)(\mathrm{b}),[\mathrm{F}(2,1448)=9.70,<0.001]($ Fig. 6d $)$ (Table 4).

Dipteran diversity demonstrated similar results to richness. Restored $(\bar{x}=0.06, \mathrm{SE}=0.01)$ (a) and reference sites $(\bar{x}=0.04, \mathrm{SE}=0.007)(\mathrm{ab})$ had higher richness than cropland sites $(\bar{x}=0.02, \mathrm{SE}=0.006)(\mathrm{b}),[\mathrm{F}(2,1448)=$
Fig. 5 Differences in Diptera abundance and richness from insect samples collected from blue vane traps in the Rainwater Basin of Nebraska in 2014 and 2015. a Abundance among three land-use conditions across 14 sampling periods. b Richness among three land-use conditions across 14 sampling periods. Crop $=$ cropland, Ref $=$ reference and $\mathrm{Res}=$ restored land-condition conditions. Bars indicate standard error a

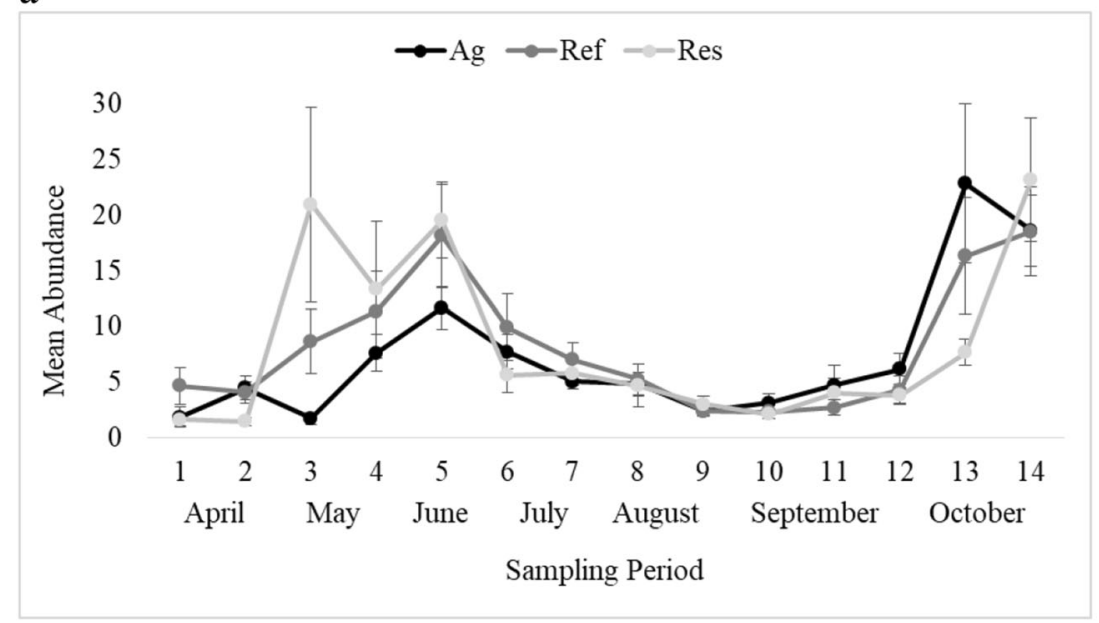

b

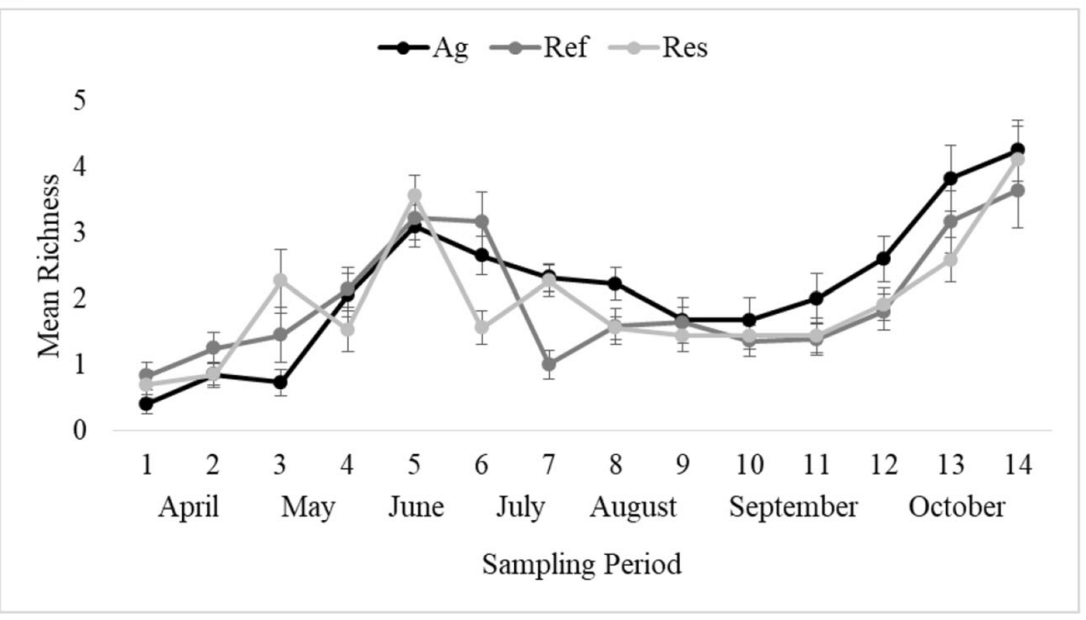


Table 3 Abundance, species richness, and diversity of Diptera collected with vane traps in the Rainwater Basin of Nebraska during the growing seasons of 2014 and 2015

\begin{tabular}{|c|c|c|c|c|c|}
\hline Source & $\mathrm{df}$ & Adj SS & Adj MS & F-value & $P$ value \\
\hline \multicolumn{6}{|l|}{ Abundance } \\
\hline Land-condition & 2 & 1004 & 501.8 & 1.69 & 0.185 \\
\hline County & 7 & 11174 & 1596.2 & 5.37 & $<0.001$ \\
\hline Period & 13 & 46517 & 3578.3 & 12.04 & $<0.001$ \\
\hline Position & 1 & 693 & 693.5 & 2.33 & 0.127 \\
\hline Year & 1 & 202 & 201.5 & 0.68 & 0.410 \\
\hline Land-condition*Period & 26 & 13011 & 500.4 & 1.68 & 0.017 \\
\hline Land-condition*Position & 2 & 1288 & 644.0 & 2.17 & 0.115 \\
\hline Position*Period & 13 & 3734 & 287.2 & 0.97 & 0.483 \\
\hline Land-condition*Position*Period & 26 & 3473 & 133.6 & 0.45 & 0.993 \\
\hline Error & 1420 & 422132 & 297.3 & & \\
\hline Lack of fit & 944 & 276519 & 292.9 & 0.96 & 0.711 \\
\hline Pure error & 476 & 145612 & 305.9 & & \\
\hline Total & 1511 & 502763 & & & \\
\hline \multicolumn{6}{|l|}{ Richness } \\
\hline Land-condition & 2 & 7.57 & 3.785 & 1.04 & 0.355 \\
\hline County & 7 & 129.31 & 18.473 & 5.06 & $<0.001$ \\
\hline Period & 13 & 1215.17 & 93.475 & 25.58 & $<0.001$ \\
\hline Position & 1 & 0.54 & 0.541 & 0.15 & 0.700 \\
\hline Year & 1 & 249.02 & 249.020 & 68.14 & $<0.001$ \\
\hline Land-condition*Period & 26 & 162.31 & 6.243 & 1.71 & 0.015 \\
\hline Land-condition*Position & 2 & 8.20 & 4.099 & 1.12 & 0.326 \\
\hline Position*Period & 13 & 46.36 & 3.566 & 0.98 & 0.473 \\
\hline Land-condition*Position*Period & 26 & 47.18 & 1.815 & 0.50 & 0.984 \\
\hline Error & 1420 & 5189.21 & 3.654 & & \\
\hline Lack of fit & 944 & 3731.38 & 3.953 & 1.29 & 0.001 \\
\hline Pure error & 476 & 1457.83 & 3.063 & & \\
\hline Total & 1511 & 7089.33 & & & \\
\hline \multicolumn{6}{|l|}{ Diversity } \\
\hline Land-condition & 2 & 1.048 & 0.5239 & 2.17 & 0.115 \\
\hline County & 7 & 6.995 & 0.9992 & 4.13 & $<0.001$ \\
\hline Period & 13 & 60.823 & 4.6787 & 19.34 & $<0.001$ \\
\hline Position & 1 & 0.033 & 0.0328 & 0.14 & 0.713 \\
\hline Year & 1 & 28.683 & 28.6831 & 118.56 & $<0.001$ \\
\hline Land-condition*Period & 26 & 11.258 & 0.4330 & 1.79 & 0.009 \\
\hline Land-condition*Position & 2 & 0.384 & 0.1919 & 0.79 & 0.453 \\
\hline Position*Period & 13 & 1.842 & 0.1417 & 0.59 & 0.867 \\
\hline Land-condition*Position*Period & 26 & 3.693 & 0.1420 & 0.59 & 0.951 \\
\hline Error & 1420 & 343.544 & 0.2419 & & \\
\hline Lack of fit & 944 & 246.172 & 0.2608 & 1.27 & 0.001 \\
\hline Pure error & 476 & 97.372 & 0.2046 & & \\
\hline Total & 1511 & 460.509 & & & \\
\hline
\end{tabular}

ANOVA analyses were used to compare three fixed factors (land-condition type, landscape position, and sampling period) and two random factors (year and county)
$7.38,0.001]$. Playas in period $11(x=0.31, \mathrm{SE}=0.08)(\mathrm{a})$ had higher diversity than upland sites $(x=0.0, \mathrm{SE}=0.0)$ (c), $[\mathrm{F}(13,1448)=7.12, \mathrm{p}=<0.001]$. Playas in period 10 $(\bar{x}=0.60, \mathrm{SE}=0.15)$ were more diverse than upland sites $(\bar{x}=0.14, \mathrm{SE}=0.01),[\mathrm{F}(13,1448)=7.12,<0.001]$ (Table 4). 
a

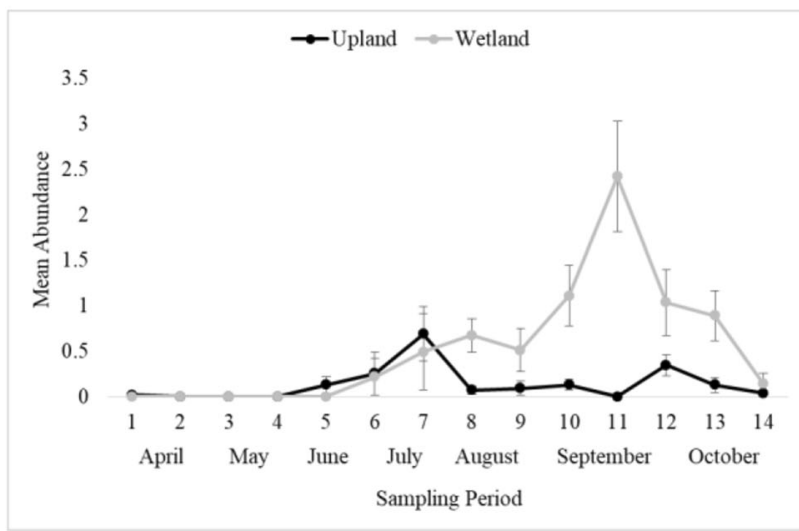

C

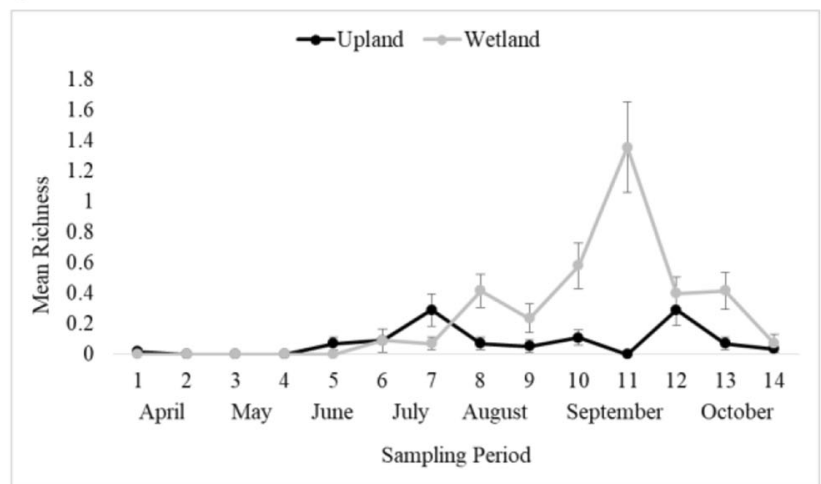

Fig. 6 Differences in Diptera abundance and richness from targeted net samples collected in the Rainwater Basin of Nebraska in 2014 and 2015. a Two-way interaction of land position*sampling period over 14 sampling periods. b Abundance among three land-use conditions. c b

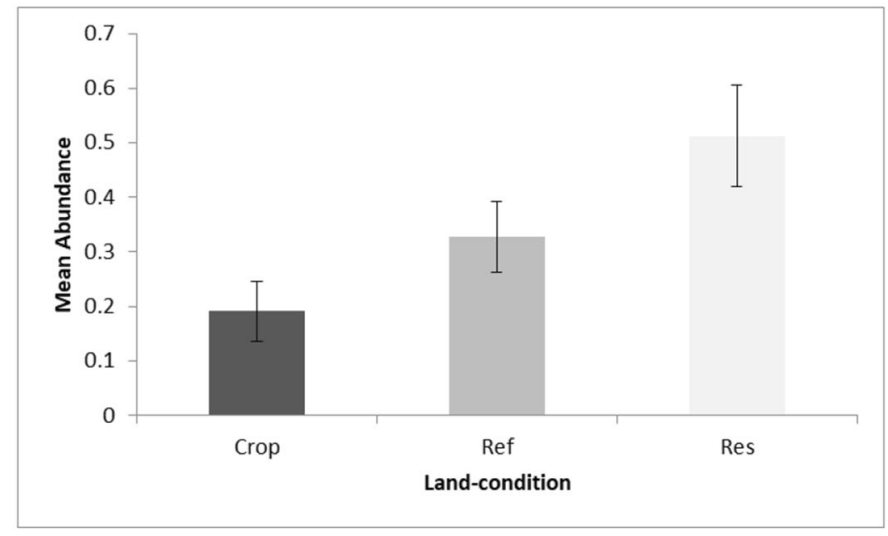

d

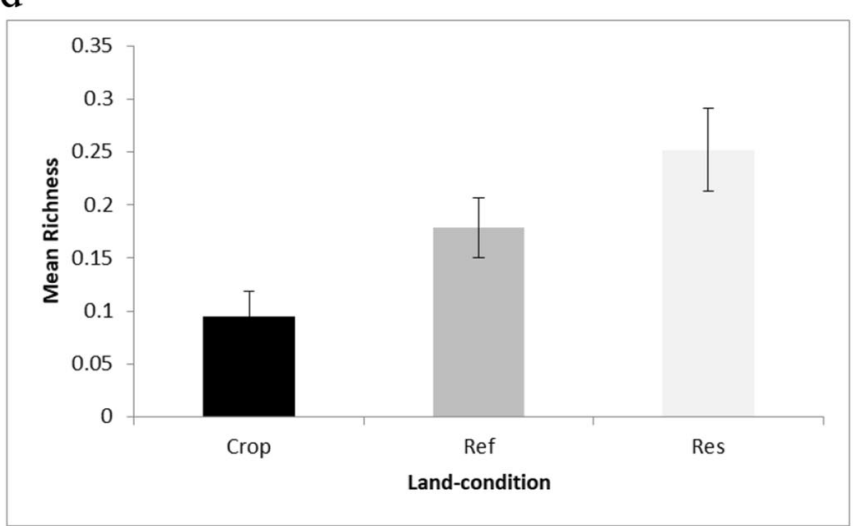

Two-way interaction of land position*sampling period over 14 sampling periods. $\mathbf{d}$ Richness among three land-use conditions. Crop = cropland

\section{Discussion}

\section{Hymenoptera}

When we examined vane trap data, there were no strong patterns of differences in Hymenoptera abundance, richness and diversity among land-condition types and among playas and upland plant communities. Reference uplands had a greater abundance of hymenopterans than cropped uplands, but hymenopteran abundance in reference uplands was not statistically different from their abundance in restored uplands or playas in all land-condition types. All playas, regardless of land use, had similar hymenopteran abundance, richness, and diversity to adjacent uplands in trap collections, suggesting that surrounding land-condition does not affect, or only minimally affects, use of a playas by bees. Similar studies have conflicting results. Evans et al. (2018) determined that wetland presence enhanced wild bee abundance and richness in agricultural landscapes. Mogren et al. (2016), however, found that if wetlands surrounding

agricultural lands were in a degraded condition, wildpollinator abundance and richness did not differ from surrounding land-conditions. We also need to consider capture methods. Vane traps are powerful attractants (Stephen and Rao 2007) and could be drawing in bees that would otherwise move to other foraging areas. Our data did support that wild-pollinator richness at cropland sites was lower than at reference and restoration sites, which is consistent with findings that habitat loss is a major contributor to pollinator decline (Winfree et al. 2009) and distance from natural areas results in reduced pollinator richness (Ricketts et al. 2008). However, these results also showed that playas in all landcondition types were statistically similar to cropland. Grasslands are an important habitat for wild bees (Kimoto et al. 2012). Therefore, although wetlands in all landcondition types had similar abundances of bees, grassland watersheds may be providing additional resources (i.e., nesting habitat) (Westrich 1996; Hoffman Black et al. 2011) for a wider array of bee species that are not found in or utilizing wetlands. Although one of the goals of our 
Table 4 Abundance, species richness, and diversity of Diptera collected with net sampling in the Rainwater Basin of Nebraska during the growing seasons of 2014 and 2015

\begin{tabular}{|c|c|c|c|c|c|}
\hline Source & $\mathrm{df}$ & Adj SS & Adj MS & $\begin{array}{l}\mathrm{F}- \\
\text { value }\end{array}$ & $P$ value \\
\hline \multicolumn{6}{|l|}{ Abundance } \\
\hline Land-condition & 2 & 33.83 & 16.917 & 7.27 & 0.001 \\
\hline County & 7 & 17.04 & 2.434 & 1.05 & 0.397 \\
\hline Period & 13 & 183.88 & 14.145 & 6.08 & $<0.001$ \\
\hline Position & 1 & 63.02 & 63.023 & 27.07 & $<0.001$ \\
\hline Year & 1 & 2.84 & 2.840 & 1.22 & 0.270 \\
\hline Land-condition*Period & 26 & 84.04 & 3.232 & 1.39 & 0.093 \\
\hline Land-condition*Position & 2 & 6.39 & 3.194 & 1.37 & 0.254 \\
\hline Position*Period & 13 & 170.05 & 13.081 & 5.62 & $<0.001$ \\
\hline Land-condition*Position*Period & 26 & 33.38 & 1.284 & 0.55 & 0.968 \\
\hline Error & 1448 & 3371.42 & 2.328 & & \\
\hline Lack of fit & 972 & 2540.26 & 2.613 & 1.50 & $<0.001$ \\
\hline Pure error & 476 & 831.17 & 1.746 & & \\
\hline Total & 1539 & 3957.11 & & & \\
\hline \multicolumn{6}{|l|}{ Richness } \\
\hline Land-condition & 2 & 7.796 & 3.8978 & 9.70 & $<0.001$ \\
\hline County & 7 & 4.146 & 0.5922 & 1.47 & 0.172 \\
\hline Period & 13 & 54.929 & 4.2253 & 10.52 & $<0.001$ \\
\hline Position & 1 & 13.142 & 13.1421 & 32.71 & $<0.001$ \\
\hline Year & 1 & 0.640 & 0.6398 & 1.59 & 0.207 \\
\hline Land-condition*Period & 26 & 10.291 & 0.3958 & 0.99 & 0.485 \\
\hline Land-condition*Position & 2 & 1.146 & 0.5731 & 1.43 & 0.240 \\
\hline Position*Period & 13 & 55.271 & 4.2516 & 10.58 & $<0.001$ \\
\hline Land-condition*Position*Period & 26 & 5.267 & 0.2026 & 0.50 & 0.982 \\
\hline Error & 1448 & 581.737 & 0.4018 & & \\
\hline Lack of fit & 972 & 447.237 & 0.4601 & 1.63 & $<0.001$ \\
\hline Pure error & 476 & 134.500 & 0.2826 & & \\
\hline Total & 1539 & 732.085 & & & \\
\hline \multicolumn{6}{|l|}{ Diversity } \\
\hline Land-condition & 2 & 0.4801 & 0.24003 & 7.38 & 0.001 \\
\hline County & 7 & 0.2470 & 0.03528 & 1.08 & 0.371 \\
\hline Period & 13 & 2.7962 & 0.21509 & 6.61 & $<0.001$ \\
\hline Position & 1 & 0.7344 & 0.73443 & 22.57 & $<0.001$ \\
\hline Year & 1 & 0.0783 & 0.07834 & 2.41 & 0.121 \\
\hline Land-condition*Period & 26 & 0.7859 & 0.03023 & 0.93 & 0.567 \\
\hline Land-condition*Position & 2 & 0.1472 & 0.07359 & 2.26 & 0.105 \\
\hline Position*Period & 13 & 3.0103 & 0.23156 & 7.12 & $<0.001$ \\
\hline Land-condition*Position*Period & 26 & 0.6530 & 0.02512 & 0.77 & 0.787 \\
\hline Error & 1448 & 47.1111 & 0.03254 & & \\
\hline Lack of fit & 972 & 34.9022 & 0.03591 & 1.40 & $<0.001$ \\
\hline Pure error & 476 & 12.2089 & 0.02565 & & \\
\hline Total & 1539 & 55.8758 & & & \\
\hline
\end{tabular}

ANOVA analyses were used to compare three fixed factors (land-condition type $<0.001$, landscape position, and sampling period) and two random factors (year and county) study was to determine the value of playas to hymenoptera pollinators individually, their value may be more important as part of the landscape matrix (Vickruck et al. 2019).
Net captures tell a different story from trap samples, and this is consistent with results from Meyer et al. (2017). These data suggest that bees forage more in playas than uplands in July 
and August. However, in September, bees foraged more in adjacent uplands, suggesting bees shift foraging areas as food supplies change. For example, a greater abundance of bees captured in playas than uplands during mid-summer was because of foraging on wetland plants such as smartweed (Polygonum spp. and Persicaria spp.) and plains coreopsis (Coreopsis tinctoria) (Park 2017). Foraging later in the season shifted to surrounding uplands to take advantage of late-season forbs (e.g. goldenrods [Solidago spp. and sunflowers [Helianthus spp.]) blooming in the uplands during late summer (Park 2017). Vickruck et al. (2019) determined that wetlands were an important seminatural habitat feature in the Prairie Pothole Region, and supported wild bee populations that were distinct. The targeted netting data allowed us a more specific window into how pollinators are using uplands and playas differently, and that playas may be distinctly important for some bee populations.

\section{Diptera}

Although flies only comprised $12 \%$ of the trap data, they had the most individuals of any order in net collections (32\%). The largest Dipteran family was Syrphidae, or hoverflies - a Hymenoptera mimicker and an efficient pollinator in temperate zones (Willmer 2011). Syrphid flies are recognized pollinators (Willmer 2011), and can use a variety of habitats (Kearns 2001), and playas can provide additional resources not as readily available in grasslands alone. Hoverflies from the tribes Eristalini and Sericomyiini deposit eggs in water and the larvae are aquatic (Vockeroth and Thompson 1987), Five genera from Eristalini were represented in our trap samples. Hoverfly larvae use rotting organic matter in wetlands that can be provided by playas (Gilbert 1986; Vockeroth and Thompson 1987). In trap collections, flies were more abundant in playas than in uplands. This was also observed in net collections, especially during mid-summer. This indicates that flies are utilizing habitat in proximity of the playas, enhancing the scope of pollinating insects in the landscape.

Net and trap abundance, richness, and diversity metrics varied throughout the season. However, playas typically had higher abundance of flies than uplands. Abundance and richness peaked in May/early June, declined in summer, and then increased again in September. Syrphids annually produce two or three generations (Gilbert 1986). The peaks in abundance and richness may be due to generations of larvae hatching in late spring and fall when playas typically have more water present. The increase could also be due to different species emerging at various times throughout the season.

Although playas, even when surrounded by crop fields, can provide reproductive sites for hoverflies, pollen and nectar producing forbs are still required to complete their life cycle. Therefore, promoting forb production by removing sediment from playas and planting forb-rich buffer strips can assist resilient pollinators, such as hoverflies, to persist in an agriculturally dominated landscape.

\section{Recommendations}

Although our data did not show strong evidence of differences among land-condition types and between playas and uplands, it did indicate that reference and restored sites support greater richness of hymenopterans, and playas are likely providing additional habitat resources for dipterans and additional floral resources for both orders. This is consistent with research that has demonstrated the importance of natural areas for wild bees (Garibaldi et al. 2011; Smith et al. 2013; Alomar, 2018) and dipterans (Kearns 2001). Playas in the RWB are the primary remaining natural habitats available to provide necessary resources to pollinators to complete their life history. However, playas are a small fraction of the landscape - approximately $0.4 \%$ (Walker 2016). Conserving these wetland areas could be beneficial to both hymenopteran and dipteran pollinators.

The WRP has been critical in protecting and restoring the few remaining playas in the RWB. Restoration practices for playas such as sediment removal are important for restoring the natural hydroperiod, removing invasive species, and allowing the native seed bank to establish (Beas et al. 2013), thus providing more forage for pollinators. Furthermore, restoring adjacent uplands could be helpful to pollinators, especially for bees that need grasslands for nest sites, nest material, and overwintering habitat (Westrich 1996; Hoffman Black et al. 2011). This is also important in late season when many pollinators use the adjacent uplands.

In the agricultural matrix of the RWB, playas and adjacent uplands are providing important habitat (i.e. food, nesting resources, and overwintering sites) for bees. In order to maintain the ecosystem service of flowering-plant pollination, it is critical to promote programs such as the WRP (now WRE), which are restoring playas through watershed development, diverse forb communities, and enhancement of wetland seed banks through sediment removal (Beas et al. 2013). Playas void of buffer strips are beneficial for habitat generalists such as hoverflies, but lack the necessary resources to sustain bee populations. Continuing to protect and enhance the entire playa ecosystem, including the watershed, is essential to maintaining natural or semi-natural areas to protect the full diversity of pollinators in the RWB and support pollination services.

Acknowledgements Funding for this project was provided the U.S. Environmental Protection Agency Region VII Office and Oklahoma State University. Additional support was provided by USDA CEAP Wetlands. We thank Lydia Horne, Dylan Cleary, and Sam Stroebel for field assistance. The Nebraska Game and Parks Commission provided field vehicles and housing. Randy Stutheit, Gerry Steinauer, and Bob Meduna provided expertise and assistance with logistics. We also thank Kris Giles for earlier reviews of this material. Lisa Overall provided essential pollinator identification assistance.

Open Access This article is distributed under the terms of the Creative Commons Attribution 4.0 International License (http:// 
creativecommons.org/licenses/by/4.0/), which permits unrestricted use, distribution, and reproduction in any medium, provided you give appropriate credit to the original author(s) and the source, provide a link to the Creative Commons license, and indicate if changes were made.

\section{References}

Aizen MA, Sabatiano M, Tylianakis JM (2012) Specialization and rarity predict nonrandom loss of interactions from mutualistic networks. Science 335:1486-1489

Alomar D, González-Estévez M, Traveset A, Lázaro A (2018) The intertwined effects of natural vegetation, local flower community, and pollinator diversity on the production of almond trees. Agriculture, Ecosystems \& Environment 264:34-43

Beas BJ, Smith LM, LaGrange TG, Stutheit R (2013) Effects of sediment removal on vegetation communities in Rainwater Basin playa wetlands. Journal of Environmental Management 128:371-379

Biesmeijer JC, Roberts SP, Reemer M, Ohlemüller R, Edwards M, Peeters T, Settele J (2006) Parallel declines in pollinators and insect-pollinated plants in Britain and the Netherlands. Science 313:351-354

Black SH, Shepherd M, Vaughan M (2011) Rangeland management for pollinators. Rangelands 33(3):9-13

Brittain C, Kremen C, Klein AM (2013) Biodiversity buffers pollination from changes in environmental conditions. Global Change Biology 19(2):540-547

Calderone N (2012a) Insect pollinated crops, insect pollinators and US agriculture: trend analysis of aggregate data for the period 19922009. PLoS One 7(5):e37235

Calderone N (2012b) The contribution of insect pollinators to U.S. agriculture. Bee Culture 140(12):32-38

Delaplane KS, Mayer DF (2000) Crop pollination by bees. CABI Publishing, Wallingford

Eeraerts M, Meeus I, Van Den Berge S, Smagghe G (2017) Landscapes with high intensive fruit cultivation reduce wild pollinator services to sweet cherry. Agriculture, Ecosystems and Environment 239:342-348

Evans E, Smart M, Cariveau D, Spivak M (2018) Wild, native bees and managed honeybees benefit from similar agricultural land uses. Agriculture, Ecosystems and Environment 268:162-170

Fründ J, Linsenmair KE, Blüthgen N (2010) Pollinator diversity and specialization in relation to flower diversity. Oikos 119:1581-1590

Garibaldi, L. A., Steffan-Dewenter, I., et al. (2011) Stability of pollination services decreases with isolation from natural areas despite honeybee visits. Ecology Letters 14(10): 1062-1072

Garibaldi, L. A., Steffan-Dewenter, I., et al. (2013) Wild pollinators enhance fruit set of crops regardless of honeybee abundance. Science 339(6127): 1608-1611

Gathmann A, Tscharntke T (2002) Foraging ranges of solitary bees. Journal of Animal Science 71:757-764

Gibbs J, Joshi NK et al (2017) Does passive sampling accurately reflect the bee (Apoidea: Anthophila) communities pollinating apple and sour cherry orchards? Environment and Ecology 46(3):579-588

Gilbert FS (1986) Hoverflies. Cambridge University Press, Cambridge

Gleason RA, Euliss NH, Tangen BA, Laubhan MK, Browne BA (2011) USDA conservation program and practice effects on wetland ecosystem services in the Prairie Pothole Region. Ecological Applications 21:S65-S81

Goulson D, Nicholls E (2016) The canary in the coalmine; bee declines as an indicator of environmental health. Science Progress 99(3):312-326

Grosse R (2014) Number and ownership of Rainwater Basin wetlands. Message to C. Park. 13 August 2014. E-mail. Rainwater Basin Joint Venture. Grand Island, NE, USA

Guthery FS, Stormer FA (1984) Wildlife management scenarios for playa vegetation. Wildlife Society Bulletin 12:227-234
Hall M (2018) Blue and yellow vane traps differ in their sampling effectiveness for wild bees in both open and wooded habitats. Agricultural and Forest Entomology 20:487-495

Hatfield R, Jepsen S, Mader E, Black SH, Shephard M (2012) Conserving bumble bees: guidelines for creating and managing habitat for Americas declining pollinators. Xerces Society for Invertebrate Conservation, Portland

Haukos DA, Smith LM (1994) The importance of playa wetlands to biodiversity of the Southern High Plains. Landscape and Urban Planning 28:83-98

Haukos DA, Smith LM (2003) Past and future impacts of wetland regulations on playa ecology in the Southern Great Plains. Wetlands 23: 577-589

Holzschuh A, Dudenhöffer JH, Tscharntke T (2012) Landscapes with wild bee habitats enhance pollination, fruit set and yield of sweet cherry. Biological Conservation 153:101-107

Inouye D, Larson BMH, Kevan P (2015) Flies and flowers III: ecology of foraging and pollination. J Pollinat Ecol 16(16):115-133

James R, Pitts-Singer TL (2008) Bee pollination in agricultural ecosystems. Oxford University Press, Oxford

Jauker F, Diekötter T, Schwarzbach F, Wolters V (2009) Pollinator dispersal in an agricultural matrix: opposing responses of wild bees and hoverflies to landscape structure and distance from main habitat. Landscape Ecology 24:547-555

Kearns C (2001) North American dipteran pollinators: assessing their value and conservation status. Conservation Ecology 5:1-13

Kennedy, C. M., Lonsdorf, E., et al. (2013) A global quantitative synthesis of local and landscape effects on wild bee pollinators in agroecosystems. Ecology Letters 16: 584-599

Kimoto C, DeBano SJ, Thorp RW, Rao S, Stephen WP (2012) Investigating temporal patterns of a native bee community in a remnant North American bunchgrass prairie using blue vane traps. Journal of Insect Science 12:108

Klein AM, Vaissière BE, Cane JH, Steffan-Dewenter I, Cunningham SA, Kremen C, Tscharntke T (2007) Importance of pollinators in changing landscapes for world crops. Proceedings of the Royal Society of London - Series B: Biological Sciences 274:303-313

Koh I, Lonsdorf EV, Williams NM, Brittain C, Isaacs R, Gibbs J, Ricketts TH (2016) Modeling the status, trends, and impacts of wild bee abundance in the United States. Proceedings of the National Academy of Sciences of the United States of America 113(1):140-145

Kratschmer S, Pachinger B et al (2018) Tillage intensity or landscape features: what matter most for wild bee diversity in vineyards? Agriculture, Ecosystems \& Environment 266:142-152

Kuchler AW (1964) Potential natural vegetation of the conterminous United States, vol 36. American Geographical Society, New York

Kulhanek K, Steinhaur N et al (2017) A national survey of managed honeybee 2015-2016 annual colony losses in the USA. Journal of Apicultural Research 56(4):328-340

Kwaiser KS, Hendrix SD (2007) Diversity and abundance of bees (Hymenoptera: Apiformes) in native and ruderal grasslands of agriculturally dominated landscapes. Agriculture, Ecosystems and Environment 124:200-2004

LaGrange T (2005) Guide to Nebraska's wetlands and their conservation needs. Nebraska Game and Parks Commission, Lincoln

LaGrange TG, Stutheit R, Gilbert M, Shurtliff D, Whited PM (2011) Sedimentation of Nebraska's playa wetlands: a review of current knowledge and issues. Nebraska Game and Parks Commission, Lincoln

Larson DL (2008) Invasive plants and pollinator interactions. Endanger Species Bull 25:46-48

Larson BMH, Kevan P, Inouye D (2001) Flies and flowers: taxonomic diversity of anthophiles and pollinators. Canadian Entomologist 133:439-465

M'Gonigle LK, Ponisio LC, Cutler K, Kremen C (2015) Habitat restoration promotes pollinator persistence and colonization in intensively managed agriculture. Ecological Applications 25(6):1557-1565 
Mallinger RE, Gratton C (2015) Species richness of wild bees, but not the use of managed honeybees, increases fruit set of a pollinatordependent crop. Journal of Applied Ecology 52:323-330

McKechnie IM, Thomsen CJM, Sargent RD (2017) Forested field edges support a greater diversity of wild pollinators in lowbush blueberry (Vaccinium angustifolium). Agriculture, Ecosystems and Environment 237(16):154-161

Meyer S, Unternahrer D, Arlettaz R, Humbert JY, Menz M (2017) Promoting diverse communities of wild bees and hoverflies requires a landscape approach to managing meadows. Agriculture, Ecosystems \& Environment 239:376-384

Mogren CL, Rand TA, Fausti SW, Lundgren J (2016) The effects of crop intensification on the diversity of native pollinators. Environmental Entomology 45(4):865-872

Natural Resource Conservation Service (NRCS) (2008a) At a glance: wetlands reserve program. United States Department of Agriculture, Washington, DC

Natural Resource Conservation Service (NRCS) (2008b) The wetlands reserve program supports migrating waterfowl in Nebraska's Rainwater Basin region. United States Department of Agriculture, Lincoln

O'Connell J, Johnson L, Smith LM, McMurry ST, Haukos DA (2012) Influence of land-use and conservation programs on wetland plant communities of the semi-arid United States Great Plains. Biological Conservation 146:108-115

Orford KA, Vaughn IP, Memmott J (2015) The forgotten flies: the importance of non-syrphid Diptera as pollinators. Proceedings of the Royal Society B: Biological Sciences 282:20142934

Park CN (2017) Evaluating how wetland presence and restoration effects landscape and resource use of pollinator communities in an agricultural matrix. MS Thesis, Oklahoma State University, Stillwater, OK, USA

Park CN, Overall LM, Smith LM, LaGrange T, McMurry ST (2017) Melittofauna and other potential pollinators in wetland and uplands in south central Nebraska (Insecta: Apoidea). Zootaxa. 4242:255-280

Potts SG, Vuilliamy SR, Roberts S, O'Toole C, Dafni A, Ne'eman G, Willmer P (2005) Role of nesting resources in organizing diverse bee communities in a Mediterranean landscape. Ecological Entomology 30:78-85

Potts SG, Biesmeijer JC, Kremen C, Neumann P, Schweiger O, Kunin WE (2010) Global pollinator declines: trends, impacts and drivers. Trends in Ecology \& Evolution 25:345-353

Rader R, Bartomeus I, Garibaldi LA, Garratt MP, Howlett BG, Winfree R, Bommarco R (2016) Non-bee insects are important contributors to global crop pollination. Proceedings of the National Academy of Sciences 113:146-151

Rainwater Basin Joint Venture (2019) About the Rainwater Basin. http:// rwbjv.org/rainwater-basin-joint-venture/about-the-rainwater-basin/. Accessed 08 Sept 2019

Rainwater Basin Joint Venture Public Lands Workgroup (2016) Best management practices for Rainwater Basin Wetlands. Rainwater Basin Joint Venture Report, Grand Island

Rhoades P, Griswold T, Waits L, Bosque-Pérez NA, Kennedy CM, Eigenbrode SD (2017) Sampling techniques affects detection of habitat factors influencing wild bee communities. Journal of Insect Conservation 21:703-714

Rhodes CJ (2018) Pollinator decline - an ecological calamity in the making? Science Progress 101(2):121-160

Ricketts TH, Regetz J, Steffan-Dewenter I, Cunningham SA, Kremen C, Bogdanski A, Gemmill-Herren B, Greenleaf SS, Klein AM, Mayfield MM, Morandin LA, Ochieng' A, Viana BF (2008) Landscape effects on crop pollination services: are there general patterns? Ecology Letters 11:499-515

Samson F, Knopf F (1994) Prairie conservation in North America. BioScience. 44:418-421
Skevington JH, Locke MM, Young AD, Moran K, Crins WJ, Marshall SA (2019) Field guide to the flower flies of northeastern North America. Princeton University Press, Princeton

Smith LM (2003) Playas of the Great Plains. University of Texas Press, Austin

Smith LM, Haukos DA, McMurry ST, LaGrange T, Willis D (2011) Ecosystem services provided by playas in the High Plains: potential influences of USDA conservation programs. Ecological Applications 21:S82-S92

Smith AA, Bentley M, Reynolds HL (2013) Wild bees visiting cucumber on Midwestern U.S. organic farms benefit from near-farm seminatural areas. Journal of Economic Entomology 106(1):97-106

Ssymank A, Kearns CA, Pape T, Thompson FC (2008) Pollinating flies (Diptera): A major contribution to plant diversity and agricultural production. Biodiversity 9(1-2):86-89

Steffan-Dewenter I, Tscharntke T (1999) Effects of habitat isolation on pollinator communities and seed set. Oecologia 121:432-440

Stephen WP, Rao S (2005) Unscented color traps for non-Apis bees (Hymenoptera: Apiformes). Journal of the Kansas Entomological Society 78:373-380

Stephen WP, Rao S (2007) Sampling native bees in proximity to a highly competitive food resource. Journal of the Kansas Entomological Society 80:369-376

Stokstad E (2007) The case of the empty hives. Science 316:970-972

Stutheit RG, Gilbert MC, Whited PW, Lawrence KL (2004) A regional guidebook for applying the hydrogeomorphic approach to assessing wetland functions of rainwater basin depressional wetlands in Nebraska. U.S. Corps of Engineers, Omaha

Triplehorn CAJ, Borror NF, Triplehorn DJ, Johnson NF (2005) Borror and DeLong's introduction to the \study of insects. Thompson Brooks/Cole, Belmont

vanEngelsdorp D, Evans JD, Saegerman C, Mullin C, Haubruge E, Nguyen BK, Frazier M, Frazier J, Cox-Foster D, Chen Y, Underwood R, Tarpy DR, Pettis JS (2009) Colony collapse disorder: a descriptive study. PLoS One 4:e6481

Vickruck JL, Best LR, Gavin MP, Devries PG (2019) Pothole wetlands provide reservoir habitat for native bees in prairie croplands. Biological Conservation 232:43-50

Vockeroth JR, Thompson FC (1987) Syrphidae. In: McAlpine JF, Peterson BV, Shewell GE, Teskey HJ, Vockeroth JR, Wood DM (eds) Manual of Nearctic Diptera volume 2. Research Branch Agriculture Canada, Ottawa, pp 731-744

Walker N (2016) Current acreage enrolled in WRP in the Rainwater Basin. Message to C. Park. 16 Feb. 2016. E-mail. Natural Resource Conservation Service. Lincoln, NE, USA

Webb EB, Smith LM, Vrtiska MP, LaGrange TG (2010) Effects of local and landscape variables on wetland bird habitat use during migration through the Rainwater Basin. Journal of Wildlife Management 74: 09-119

Weiner CN, Werner M, Linsenmair KE, Blüthgen N (2014) Land use impacts on plant-pollinator networks; interaction strength and specialization predict pollinator declines. Ecology. 95(2):466-474

Westrich P (1996) Habitat requirements of central European bees and the problems of partial habitats. Linnean Society Symposium Series 18: $1-16$

Williams NM, Crone E, Roulston TH, Minckley RL, Packer L, Potts SG (2010) Biological Conservation 143(10):2280-2291

Willmer P (2011) Pollination and floral ecology. Princeton University Press, Princeton

Winfree R, Aguilar R, Vasquez DP, Lebuhn G, Aizen MA (2009) A metaanalysis of bee's response to anthropogenic disturbance. Ecology 90(8):2068-2076

Publisher's Note Springer Nature remains neutral with regard to jurisdictional claims in published maps and institutional affiliations. 